\title{
Genes for the synthesis of the osmoprotectant glycine betaine from choline in the moderately halophilic bacterium Halomonas elongata DSM 3043
}

\author{
David Cánovas, ${ }^{1,2}+$ Carmen Vargas, ${ }^{1}$ Susanne Kneip, ${ }^{2}$ María-Jesús Morón, ${ }^{1}$ \\ Antonio Ventosa, ${ }^{1}$ Erhard Bremer ${ }^{2}$ and Joaquín J. Nieto ${ }^{1}$
}

Author for correspondence: Joaquín J. Nieto. Tel: +34 95 4556765. Fax: + 34954628162. e-mail: jjnieto@cica.es

1 Department of Microbiology and Parasitology, Faculty of Pharmacy, University of Seville, 41012 Seville, Spain

2 Laboratory for Microbiology, Department of Biology, Philipps University, Marburg D-35032, Marburg, Germany
The genes involved in the oxidative pathway of choline to glycine betaine in the moderate halophile Halomonas elongata DSM 3043 were isolated by functional complementation of an Escherichia coli strain defective in glycine betaine synthesis. The cloned region was able to mediate the oxidation of choline to glycine betaine in E. coli, but not the transport of choline, indicating that the gene(s) involved in choline transport are not clustered with the glycine betaine synthesis genes. Nucleotide sequence analysis of a $4.6 \mathrm{~kb}$ segment from the cloned DNA revealed the occurrence of three ORFs (betIBA) apparently arranged in an operon. The deduced betl gene product exhibited features typical for DNA-binding regulatory proteins. The deduced BetB and BetA proteins showed significant similarity to soluble glycine betaine aldehyde dehydrogenases and membrane-bound choline dehydrogenases, respectively, from a variety of organisms. Evidence is presented that BetA is able to oxidize both choline and glycine betaine aldehyde and therefore can mediate both steps in the synthesis of glycine betaine.

Keywords: moderate halophiles, Halomonas elongata, bet genes, osmoregulation, compatible solutes

\section{INTRODUCTION}

Moderately halophilic bacteria are defined as those prokaryotes that grow best in media containing $0.5-$ $2.5 \mathrm{M} \mathrm{NaCl}$. They constitute a very heterogeneous group of both Gram-negative and Gram-positive bacteria which are well adapted to live and thrive in high-salt environments (Ventosa et al., 1998). Among these extremophiles, Halomonas elongata displays one of the widest salinity ranges found in nature, being able to grow between $0 \cdot 1$ and $4 \mathrm{M} \mathrm{NaCl}$ (Vreeland, 1992). Apart from its important role in the ecology of hypersaline environments, this moderate halophile constitutes an excellent model to study the molecular basis of

\footnotetext{
† Present address: Departamento de Biotecnología Microbiana, Centro Nacional de Biotecnología, CSIC, Cantoblanco, 28049 Madrid, Spain.

Abbreviations: $B A D H$, glycine betaine aldehyde dehydrogenase; $C D H$, choline dehydrogenase.

The EMBL accession number for the sequence reported in this paper is AJ238780.
}

prokaryotic osmoadaptation. Moreover, it has recently received considerable interest because of its potential for use in biotechnology (Ventosa \& Nieto, 1995; Ventosa et al., 1998).

Like most other bacteria, $H$. elongata maintains its internal osmolarity and generates turgor in media of high salinity by accumulating a limited number of metabolically inert, organic compounds named compatible solutes (Ventosa et al., 1998). It is able to synthesize de novo ectoine and hydroxyectoine when grown in media lacking osmoprotectants (Cánovas et al., 1997). The genes involved in this biosynthetic pathway have recently been isolated and characterized in two H. elongata strains (Cánovas et al., 1998a ; Göller et al., 1998). This bacterium can also accumulate the compatible solute glycine betaine and structurally related osmoprotectants by transport from the external medium. It was found that glycine betaine suppressed de novo ectoine synthesis partially or completely, depending on the $\mathrm{NaCl}$ concentration in the growth medium (Cánovas et al., 1996). In addition to uptake 


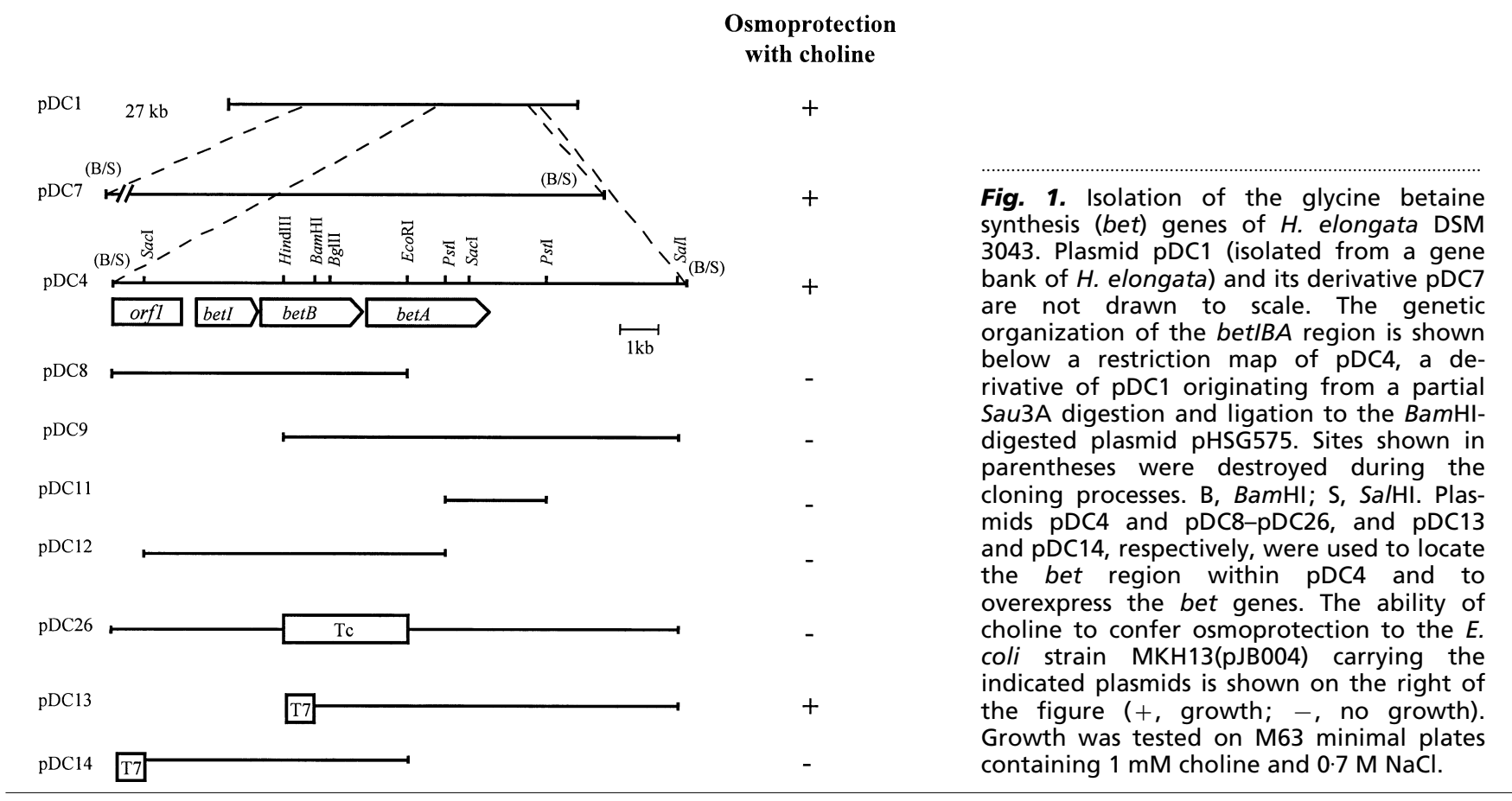

from the environment, accumulation of glycine betaine can also be achieved by oxidation from its precursor, choline (Cánovas et al., 1996). We have recently characterized a very efficient system for choline transport and its subsequent oxidation to glycine betaine. The system is mainly regulated by salinity and also by the availability of glycine betaine (Cánovas et al., 1998b). In this study, we describe the cloning, molecular characterization and expression of the genes responsible for the choline-glycine betaine biosynthetic pathway in H. elongata DSM 3043.

\section{METHODS}

Bacterials strains, media and growth conditions. Escherichia coli strains MKH13 (Haardt et al., 1995) and PD141(2DE3) (Boch et al., 1997) have been described previously. E. coli strains were routinely grown in LB medium (Miller, 1972). M63 medium (Cohen \& Rickenberg, 1956), containing 20 mM glucose as a sole carbon source, was used as minimal medium. When used, choline was added to a final concentration of $1 \mathrm{mM}$. All cultures were incubated aerobically at $37^{\circ} \mathrm{C}$ with shaking (200-220 r.p.m.). Bacterial growth was monitored spectrophotometrically at $600 \mathrm{~nm}$. Solid media contained $20 \mathrm{~g}$ Bacto-Agar (Difco) $1^{-1}$. The osmotic strength of the medium was increased by the addition of various concentrations of $\mathrm{NaCl}$. For the expression of the bet genes under the control of the T7 $\phi 10$ promoter, a modified M9 minimal medium (Miller, 1991) was used (T7 medium) containing $0 \cdot 2 \%(\mathrm{w} / \mathrm{v})$ Casamino acids, $1 \mathrm{mM} \mathrm{CaCl}_{2}, 1 \mathrm{mM} \mathrm{MgSO}_{4}$ and $0 \cdot 2 \%(\mathrm{w} / \mathrm{v})$ glucose as the carbon source. Filter-sterilized antibiotics chloramphenicol, kanamycin, ampicillin and tetracycline were added to the media at final concentrations of 30, 50, 100 and $10 \mu \mathrm{g} \mathrm{ml}{ }^{-1}$, respectively.

Conjugal transfer of plasmids. Plasmids were conjugated between $E$. coli strains by triparental matings on LB medium as described by Vargas et al. (1997) by using pRK600 (Kessler et al., 1992) as helper plasmid.

DNA manipulation and construction of plasmids. Plasmid DNA manipulations were carried out by standard techniques (Ausubel et al., 1989; Sambrook et al., 1989). The construction of a $H$. elongata DSM 3043 gene bank has been described previously (Cánovas et al., 1997). Plasmids pDC4 and pDC7 (Fig. 1) were constructed by partial digestion with Sau3AI of pDC1, a cosmid clone from the gene bank, and subsequent ligation into the BamHI-digested low-copy-number plasmid pHSG575 (Takeshita et al., 1987). pDC5 was constructed by inserting a $5.7 \mathrm{~kb} B a m \mathrm{HI}-E c o \mathrm{RI}$ fragment (carrying the E. coli betIBA genes) from pJB005 (J. Boch \& E. Bremer, unpublished data) into Bam HI /EcoRI-digested pHSG575. pDC8 and pDC9 were constructed by deleting a $3.6 \mathrm{~kb}$ EcoRI and a $2.2 \mathrm{~kb}$ HindIII fragment, respectively, from pDC4 and religation of the plasmid backbones. pDC10 and pDC11 are derivatives of the high-copy-number vector pGEM5Zf (Promega) carrying a $4.3 \mathrm{~kb}$ and a $1.3 \mathrm{~kb}$ Pst I fragment from pDC4, respectively. pDC12 was generated by deletion of a $0 \cdot 4 \mathrm{~kb}$ Sall region from pDC10. pDC13 was obtained by subcloning a $4.8 \mathrm{~kb}$ BamHISalI fragment, carrying the H. elongata bet A gene, from pDC9 into the low-copy-number expression vector pPD100 carrying the phage T7 $\phi 10$ promoter (Dersch et al., 1994). For the expression of the H. elongata betIB under the control of the same promoter, a $3.4 \mathrm{~kb}$ SacI-EcoRI fragment from pDC8 was subcloned in the polylinker of pPD101 (Dersch et al., 1994), resulting in the plasmid pDC14. To obtain pDC26, the $7 \cdot 4 \mathrm{~kb}$ SalI fragment containing the $H$. elongata betIBA region was transferred from pDC4 into the broad-host-range vector pML123 (Labes et al., 1990) to give pDC15. pDC26 was subsequently generated by inserting a Tet cassette from pOB26 (O. Schmidt-Kittler \& E. Bremer, unpublished data) between the HindIII and EcoRI sites of pDC15.

DNA sequencing of the $\boldsymbol{H}$. elongata bet region. To sequence the $H$. elongata region responsible for glycine betaine synthesis, nested unidirectional deletions of pDC12 in both orientations were generated with exonuclease III and endonuclease S1 by using the Erase-a-base kit from Promega. Resulting plasmids were named pDC16-pDC25. The DNA sequence of the betIBA region was completed by using the 
plasmids pDC4 and pDC11 and a set of synthetic oligonucleotides. Sequencing of double-stranded DNA was performed with the Thermo Sequenase fluorescence-labelled primer cycle sequencing kit with 7-deaza-dGTP (Amersham Pharmacia Biotech). The products of the sequencing reactions were separated by denaturing PAGE in a LI-COR DNA sequencer (MWG-Biotech). DNA sequence was analysed with the GCG Sequence Analysis Software Package (Genetics Computer Group) and the BLAST program of the National Center for Biotechnology Information (NCBI).

Assay for conversion of intracellular accumulated choline into glycine betaine. E. coli cells carrying the corresponding plasmids were grown to exponential phase at $37^{\circ} \mathrm{C}$ in $\mathrm{M} 63$ medium with $0.5 \mathrm{M} \mathrm{NaCl}$. Radiolabelled [methyl_ ${ }^{14} \mathrm{C}$ ]choline $(10 \mu \mathrm{M} ; 0.55 \mathrm{kBq})$ was added to the medium and the cells were incubated at room temperature for $1 \mathrm{~h}$. Samples $(0.5 \mathrm{ml})$ were taken and the cells were collected after 2 min of centrifugation in a microfuge. The cell pellet of each sample was extracted with $50 \mu \mathrm{l} 80 \%$ methanol, and glycine betaine and choline were separated by TLC on Whatman Silica Gel AL-SIL-G plates with 90:10:4 (by vol.) methanol/acetone/hydrochloric acid as the running solvent. The radioactive metabolites were visualized by autoradiography and identified by comparison with $\left[{ }^{14} \mathrm{C}\right]$ glycine betaine and $\left[{ }^{14} \mathrm{C}\right]$ choline standards.

Expression of the bet/B and bet $A$ gene products under the control of the T7 $\phi \mathbf{1 0}$ promoter. E. coli strain PD141( $\lambda \mathrm{DE} 3)$ $\left(\right.$ Bet $\left.^{-}\right)$, carrying the gene for the T7 RNA polymerase in the chromosome under lacPO control, was used as the host strain. Transconjugants of this strain containing pJB004 (E. coli bet $T$ ) plus pDC13 (H. elongata bet A), pJB004 plus pDC14 $(H$. elongata betIB) or pJB004 plasmids were grown in $\mathrm{T} 7$ medium supplemented with $0.5 \mathrm{M} \mathrm{NaCl}$ and $30 \mu \mathrm{g}$ chloramphenicol $\mathrm{ml}^{-1}$ to an $\mathrm{OD}_{600}$ of $0 \cdot 7$. The bet genes were overexpressed after induction with $1 \mathrm{mM}$ IPTG, following the procedure described by Dersch et al. (1994).

\section{RESULTS}

\section{Cloning of the genes encoding glycine betaine synthesis in $H$. elongata}

Since the genes for choline uptake (bet $T$ ) and glycine betaine synthesis (betBA) are clustered in E. coli (Lamark et al., 1991), we initially attempted to recover the $H$. elongata bet genes through functional complementation of the $\Delta$ (betTIBA) E. coli mutant strain MKH13 (Haardt et al., 1995). However, no clones were recovered that permitted the growth of MKH13 on high osmolarity minimal plates containing $1 \mathrm{mM}$ choline. Since in several other bacterial species, such as Bacillus subtilis (Boch et al., 1996) and Sinorhizobium meliloti (Pocard et al., 1997), the genes for choline transport and for the enzymic conversion of this trimethylammonium compound are not genetically linked, we adopted a cloning strategy that was successfully employed to recover the structural genes for the glycine betaine biosynthetic enzymes from B. subtilis (Boch et al., 1996). A derivative of strain MKH13 containing the E. coli choline transport gene bet $T$ on a pBR322-derived plasmid (pJB004) was used (Boch et al., 1996). Thus, strain MKH13(pJB004) can accumulate choline but it cannot convert this precursor to the osmoprotectant glycine betaine. To clone the H. elongata DSM 3043 genes responsible for glycine betaine synthesis, a gene bank of this strain constructed in the cosmid pVK102 (Cánovas et al., 1997) was transferred from E. coli HB101 to MKH13(pJB004). Transconjugants were selected on M63 plus $0.7 \mathrm{M} \mathrm{NaCl}$ and $1 \mathrm{mM}$ choline agar plates. After checking osmotolerant colonies for the presence of cosmids in addition to pJB004, eight independent colonies were found to contain three overlapping clones as judged from their restriction patterns. These clones should contain the $H$. elongata glycine betaine synthesis genes since choline does not confer osmoprotection to E. coli or B. subtilis (Landfald \& Strøm, 1986; Boch et al., 1996). Plasmid pDC1, carrying an approximately $27 \mathrm{~kb}$ insert, was selected for further characterization (Fig. 1).

To delimit the region in pDC1carrying the genes for the synthesis of glycine betaine, this plasmid was partially digested with Sau3A1 and ligated into the low-copynumber vector $\mathrm{pHSG} 575$. The resulting ligation mixture was used to transform E. coli MKH13(pJB004) and strains that were osmotolerant in the presence of choline were selected. Two plasmids, pDC4 and pDC7 (Fig. 1), containing a $7 \cdot 4 \mathrm{~kb}$ and an approximately $17 \mathrm{~kb}$ fragment from pDC1, respectively, were found to confer osmoprotection to E. coli MKH13(pJB004).

\section{Conversion of choline into glycine betaine mediated by the $H$. elongata bet genes}

To confirm that pDC1 and its derivative pDC4 conferred osmoprotection to E. coli by mediating the synthesis of glycine betaine, transconjugants of $E$. coli MKH13(pJB004) harbouring pDC1 or pDC4 were grown in $\mathrm{M} 63$ with $0.5 \mathrm{M} \mathrm{NaCl}$ to exponential-growth phase and then incubated with $10 \mu \mathrm{M}$ [methyl$\left.{ }^{14} \mathrm{C}\right]$ choline $(0.55 \mathrm{kBq})$ for $1 \mathrm{~h}$. Subsequently, the radiolabelled solutes were analysed by TLC and autoradiography. E. coli MKH13 (pJB004) carrying pDC5, a derivative of pHSG575 containing the E. coli betIBA genes, and strain MKH13(pJB004) carrying the vector pHSG575, were used as positive and negative controls, respectively. In the same experiment, E. coli MKH13 harbouring only pDC1 or pDC4 was also included to check if these plasmids were also able to mediate the transport of choline by H. elongata. As shown in Fig. 2, both pDC1 and pDC4 mediated the enzymic conversion of choline into glycine betaine when the E. coli choline transporter BetT was present. However, none of them allowed E. coli to take up choline from the medium, suggesting that in $H$. elongata the choline transport gene(s) is not linked to the glycine betaine biosynthetic genes in the chromosome.

\section{Nucleotide sequence of the bet genes}

Data presented in Fig. 2 clearly demonstrated that the $7 \cdot 4 \mathrm{~kb}$ region cloned in plasmid pDC4 encodes the enzymes necessary to oxidize exogenously provided choline to betaine. In an initial approach to map the region containing the bet genes, a number of pDC4deleted subclones (pDC8-pDC12) (Fig. 1) were constructed and tested for their ability to confer osmoprotection to E. coli MKH13(pJB004) in the presence of 


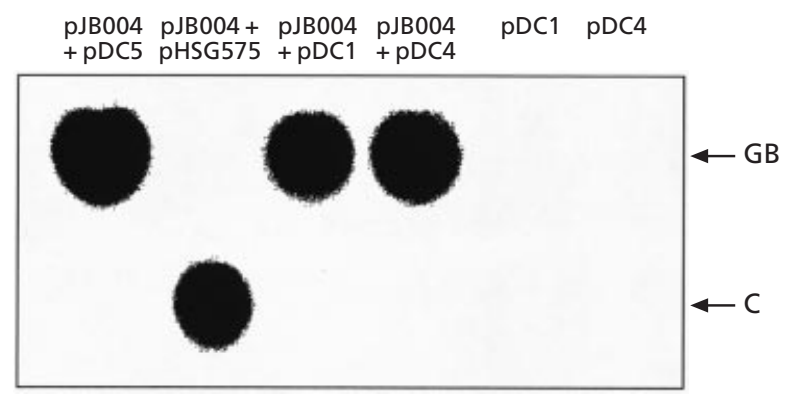

Fig. 2. Enzymic conversion of choline into glycine betaine mediated by the $H$. elongata bet genes. E. coli strain MKH13 carrying the indicated plasmids was incubated in M63 minimal medium with $0.5 \mathrm{M} \mathrm{NaCl}$ and $\left[{ }^{14} \mathrm{C}\right]$ choline for $1 \mathrm{~h}$ at room temperature. Solutes were then extracted and the radiolabelled compounds were analysed by TLC and autoradiography. Solutes were identified by comparison with $\left[{ }^{14} \mathrm{C}\right]$ glycine betaine (GB) and $\left[{ }^{14} \mathrm{C}\right]$ choline $(\mathrm{C})$ standards.

choline. None of these MKH13 derivatives could grow on high osmolarity M63 plates with choline, indicating that the $H$. elongata bet genes should lie within the central region of pDC4. This was further confirmed by replacing the HindIII-EcoRI central fragment of pDC4 by a tetracycline resistance cassette in the plasmid pDC26 (Fig. 1). This plasmid was also unable to enhance the growth of E. coli MKH13(pJB004) under high salinity conditions. Consequently, plasmids pDC4, pDC11 and pDC12 were used to determine the nucleotide sequence of a $4.6 \mathrm{~kb}$ fragment from the region cloned in pDC4.

Analysis of the DNA sequence revealed the existence of four ORFs, three complete ones (betIBA) in the same orientation and a fourth and incomplete one divergent from the others (Fig. 1). The three complete ORFs were identified as the H. elongata betIBA genes. The betI gene starts with a GTG codon at bp 659 and ends with a TAA codon at bp 1280. It encodes a 207 residue protein with a deduced molecular mass of $22 \cdot 8 \mathrm{kDa}$. The deduced amino acid sequence showed a high percentage of charged amino acids $(26 \%)$ and is rather basic, with a net positive charge of 10 . The betB gene starts with an ATG codon at bp 1293 and ends with a TAA codon at bp 2760. It encodes a 489 residue protein with a calculated molecular mass of $52 \cdot 3 \mathrm{kDa}$. The betA gene starts with an ATG codon at position 2818 and ends with a TAG codon at position 4492 . The predicted coding region encodes a 559 residue protein with a calculated molecular mass of $62.0 \mathrm{kDa}$. Divergently transcribed from the betIBA genes, the incomplete ORF (orf1) starts with an ATG codon at position 465. This ORF did not show any homology with sequences deposited in the public databases. All the ORFs were preceded by putative ribosome-binding sites. Downstream of the bet $A$ gene there is an inverted repeat which may function as a Rho-independent transcriptional terminator for the betIBA gene cluster. In addition, between positions 550 and 590 there are two sequences that display a high homology with the consensus sequences of the -10 and -35 regions of the $\sigma^{70}$ dependent promoters of E. coli.

\section{The betl gene encodes a putative regulatory protein}

Computer searches revealed a high homology of the product encoded by the H. elongata betI gene with the BetI protein of E. coli (56\% identity) and Si. meliloti (40\% identity). Moreover, a significant similarity with the $\mathrm{N}$-terminal regions of other regulatory proteins, such as the TetR repressor of plasmid pSC101 (28\% over 61 amino acids) and the MtrR repressor of Neisseria gonorrhoeae ( $27 \%$ over 63 amino acids) was found. All these homologies cover the helix-turn-helix motif typically found in DNA-binding proteins (Fig. 3a). This would suggest that the $H$. elongata betI gene encodes a regulatory protein which might, like its E. coli counterpart, serve as a repressor protein mediating bet expression in response to the availability of choline in the growth medium (Røkenes et al., 1996).

\section{BetB is a glycine betaine aldehyde dehydrogenase (BADH) homologue}

Strong homologies were found between BetB and members of the aldehyde dehydrogenase superfamily of prokaryotes and eukaryotes (Habenicht et al., 1994). The homology with the BADH from E. coli was very high $(71.4 \%$ identity). Homology was also found with BADHs from Si. meliloti $(55.5 \%)$, B. subtilis $(44.2 \%)$ and Staphylococcus xylosus $(40.6 \%$ ), and with a variety of BADHs from plants (about $40.0 \%$ ) (Fig. 3b). Moreover, the deduced BetB sequence showed $51.9 \%$ identity with the BADH from cod liver, a member of the class 9 type of BADHs for which the three-dimensional structure is known (Johansson et al., 1998). As shown in Fig. $3(\mathrm{~b})$, most of the amino acid residues belonging to the coenzyme (NAD)-binding and catalytic domains of cod liver $\mathrm{BADH}$ are conserved in the BetB protein of $H$. elongata. However, BetB lacks the leucine residue that in cod liver BADH is involved in adenosine ribose binding (Leu163 in the cod liver enzyme). In addition to cod liver $\mathrm{BADH}$, two other BADH tertiary structures have been determined, those for bovine BADH (ALDH2; Steinmetz et al., 1997) and rat BADH (ALDH3, Liu et al., 1997). In these enzymes, the adenosine ribose hydrogen bonds to a glutamic acid (Glu195 in ALDH2; Fig. 3b) which is equivalent to Glu224 in BetB. The fact that the same residue is highly conserved among all the bacterial and plant BADHs aligned in Fig. 3(b) suggests that this glutamic acid may be involved in NAD binding. From all these data it seems that the H. elongata bet $B$ gene product is a BADH.

\section{BetA is a choline dehydrogenase (CDH) homologue}

The deduced amino acid sequence of BetA showed high homology with different $\mathrm{CDH}$ s of prokaryotes, such as E. coli (74\% identity), St. xylosus (52\%) and Si. meliloti 
(a)

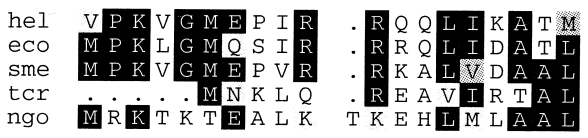

ngo
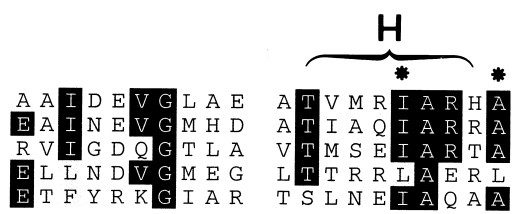
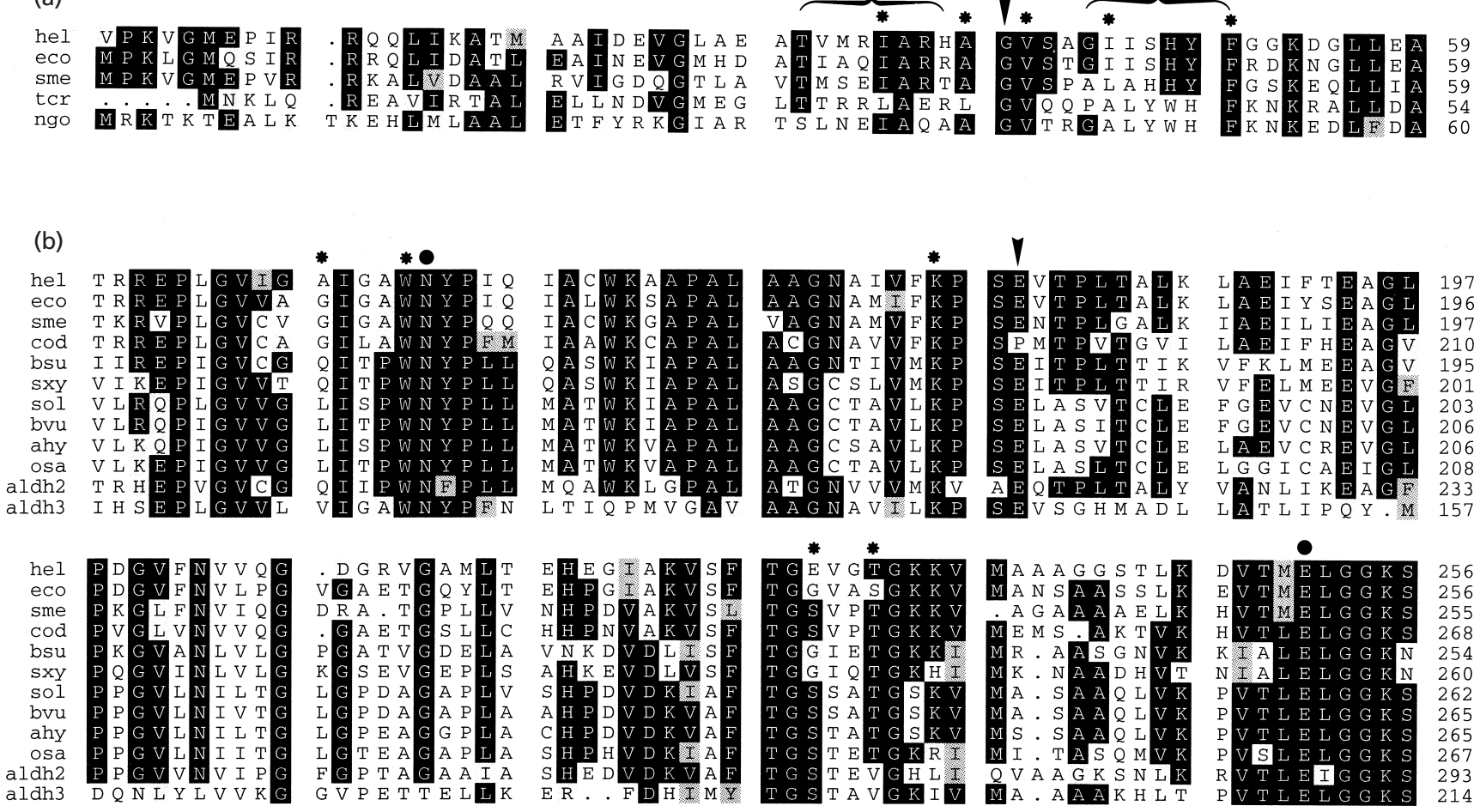

(c) hel
eco
sxy
sme
agl
rat
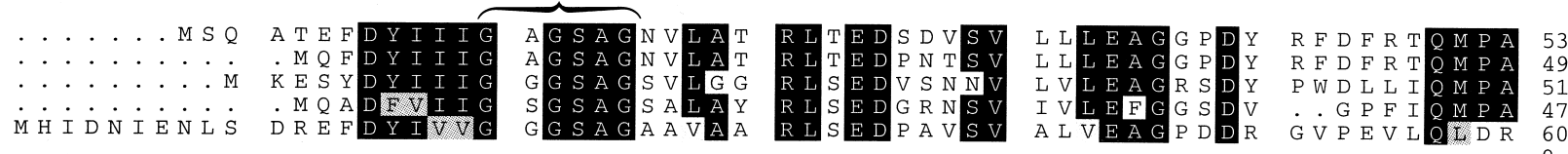

Fig. 3. Partial alignment of the deduced amino acid sequences of the $H$. elongata (hel) Betl (a), BetB (b) and BetA (c) proteins with DNA-binding regulatory proteins, aldehyde dehydrogenases and CDHs, respectively, of different organisms. (a) eco, E. coli Betl (Lamark et al., 1991); sme, Si. meliloti Betl (Østerås et al., 1998); tcr, TetR of pSC101 (Brow et al., 1985); ngo, MtrR of N. gonorrhoeae (Pan \& Spratt, 1994). The conserved helix-turn-helix (HTH) DNA-binding motif is indicated, with the conserved amino acids denoted by asterisks. (b) eco, E. coli BADH (Lamark et al., 1991); sme, Si. meliloti BADH (Pocard et al., 1997); cod, cod liver BADH (Johansson et al., 1998); bsu, B. subtilis BADH (Boch et al., 1996); sxy, St. xylosus BADH (Rosenstein et al., 1999); sol, Spinacia oleracaea BADH (Weretilnyk \& Hanson, 1990); bvu, Beta vulgaris BADH (McCue \& Hanson, 1992); ahy, Amaranthus hypochondriacus BADH (Legaria et al., 1998); osa, Oryza sativa BADH (Nakamura et al., 1997); aldh2, bovine aldehyde dehydrogenase (Guan \& Weiner, 1990); aldh3, rat aldehyde dehydrogenase (Jones et al., 1988). Asterisks indicate the residues involved in NAD binding in cod liver BADH; the arrowhead indicates the glutamic acid residue involved in NAD binding in bovine aldehyde dehydrogenase, and the dots denote the active site residues in cod liver BADH. (c) eco, E. coli CDH (Lamark et al., 1991); sxy, St. xylosus CDH (Rosenstein et al., 1999); sme, Si. meliloti CDH (Pocard et al., 1997); agl, A. globiformis choline oxidase (Deshnium et al., 1995); rat, rat CDH (Saito et al., 1997). The brace indicates the glycine box (GXGXXG) typical of flavoproteins. In all three parts of the figure, identical amino acid residues are shaded in black and conserved amino acid residues are shaded in grey. Amino acid positions are numbered on the right of the alignment.

(49\%), with the eukaryote Rattus rattus $(51 \%)$ and with the choline oxidase from Arthrobacter globiformis (31\%) (Fig. 3c). The N-terminal region of BetA displayed the so-called 'glycine box', containing a conserved motif (GXGXXG) and a series of amino acids that are characteristic features of flavoproteins (Lamark et al., 1991; Pocard et al., 1997; Wierenga et al., 1986). We concluded from these homologies that the H. elongata BetA protein might be a CDH.
The $H$. elongata BetA alone mediates the conversion of choline into glycine betaine by $E$. coli

In E. coli, the membrane-bound $\mathrm{CDH}$ encoded by the bet $A$ gene mediates not only the oxidation of choline but also the conversion of glycine betaine aldehyde into glycine betaine (Landfald \& Strøm, 1986). To check if the $H$. elongata BetA protein can also catalyse the second step of glycine betaine synthesis, the Bet $^{-}$E. coli 


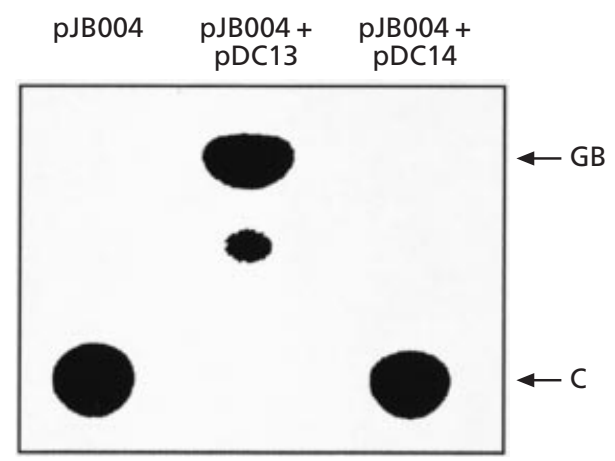

Fig. 4. The betA-encoded $\mathrm{CDH}$ is able to mediate both steps of glycine betaine synthesis. Derivatives of $E$. coli strain PD141( $\lambda D E 3)$ carrying the indicated plasmids were grown in T7 medium with $0.5 \mathrm{M} \mathrm{NaCl}$. After induction with $1 \mathrm{mM}$ IPTG for $1 \mathrm{~h}$, cells were incubated with $\left[{ }^{14} \mathrm{C}\right]$ choline for $20 \mathrm{~min}$ at room temperature. The accumulated solutes were extracted and radioactive compounds were analysed by TLC and autoradiography. Solutes were identified by comparison with $\left[{ }^{14} \mathrm{C}\right]$ glycine betaine (GB) and $\left[{ }^{14} \mathrm{C}\right]$ choline (C) standards.

strain PD141( $\lambda \mathrm{DE} 3)$ containing pJB004 (E. coli bet T) plus pDC13 (betA), pJB004 plus pDC14 (betIB), or pJB004 was used to express the corresponding proteins under the control of the $\mathrm{T} 7 \phi 10$ promoter. After induction with $1 \mathrm{mM}$ IPTG for $1 \mathrm{~h}$, cells were incubated with $\left[\right.$ methyl $\left.{ }^{14} \mathrm{C}\right]$ choline for $20 \mathrm{~min}$ at room temperature, and radiolabelled accumulated solutes were extracted and analysed by TLC. As shown in Fig. 4, only the strain carrying the bet $A^{+}$plasmid pDC13 plus the $E$. coli choline transporter gene bet $T$ converted choline into glycine betaine. An additional radioactive species might correspond to the intermediate in glycine betaine synthesis, glycine betaine aldehyde (Fig. 4). This is probably due to the short incubation time $(20 \mathrm{~min})$ with $\left[\right.$ methyl $\left.-{ }^{14} \mathrm{C}\right]$ choline used in the assay. These data strongly suggest that the $H$. elongata $\mathrm{CDH}$ is able to catalyse both steps of glycine betaine synthesis.

\section{DISCUSSION}

In addition to the synthesis of ectoines (ectoine and hydroxyectoine) (Cánovas et al., 1997, 1998a ; Göller et al., 1998), H. elongata overcomes salt stress by accumulating high amounts of glycine betaine either by transport or by synthesis (Cánovas et al., 1996; 1998b). In a previous study, we demonstrated that choline itself is not an osmoprotectant in H. elongata. Rather, choline is osmoprotective only after it is transported to the cytoplasm and enzymically transformed into glycine betaine (Cánovas et al., 1998b). In this study, we report the isolation and molecular characterization of the glycine betaine biosynthetic gene cluster (betIBA) from H. elongata DSM 3043. The isolated bet region mediates the conversion of choline by a two-step oxidation of the precursor choline involving a $\mathrm{CDH}(\mathrm{Bet} A)$ and a $\mathrm{BADH}$ (BetB). This enzymic system is not exclusive to halophilic micro-organisms. It has also been found in other
Gram-negative and Gram-positive, non-halophilic bacteria, such as E. coli (Landfald \& Strøm, 1986), Si. meliloti (Pocard et al., 1997; Østerås et al., 1998) and St. xylosus (Rosenstein et al., 1999). However, other systems can be employed for glycine betaine synthesis. For example, some Gram-positive bacteria such as $A$. globiformis use a bifunctional soluble choline oxidase (CodA; Ikuta et al., 1977; Deshnium et al., 1995). The soil bacterium $B$. subtilis uses a BADH (GbsA) in combination with a soluble type III alcohol dehydrogenase (GbsB) (Boch et al., 1996). Finally, glycine betaine synthesis in higher plants involves a BADH in combination with a choline monooxygenase (Brouquisse et al., 1989; McCue \& Hanson, 1992).

Analysis of the $H$. elongata bet region revealed the presence of three genes (betIBA), which can be functionally expressed in E. coli and seem to be genetically organized in an operon. This organization differs from that found in E. coli (betTIBA; Lamark et al., 1991), Si. meliloti (betICBA; Østerås et al., 1998), B. subtilis (gbsAB; Boch et al., 1996) and St. xylosus (cudTCAB; Rosenstein et al., 1999). Among these gene arrangements, the E. coli and St. xylosus regions carry the choline transport (bet T or $c u d T$ ) genes linked to the synthesis genes. We did not detect, either upstream or downstream of the betIAB cluster of $H$. elongata, an ORF(s) that could encode a choline transport system. Moreover, the cosmid clone pDC1, which comprises approximately $35 \mathrm{~kb}$ of the $H$. elongata chromosome, including the betIBA region, was unable to mediate the conversion of choline by E. coli strain MKH13 unless the E. coli choline transporter BetT was present. We have previously reported the existence of a high-affinity transport system for choline $\left(K_{\mathrm{m}}=10 \mu \mathrm{M}\right)$ in $H$. elongata (Cánovas et al., 1998b). This transporter must therefore be encoded elsewhere in the $H$. elongata chromosome. This situation may be similar to that found in B. subtilis, where choline uptake is mediated by two evolutionarily closely related $\mathrm{ABC}$ transport systems (OpuB and $\mathrm{OpuC}$ ), whose genes are separated from the glycine betaine biosynthetic gene locus ( $g b s A B)$ (Kappes et al., 1999).

The betICBA operon in Si. meliloti includes a gene encoding a choline sulfatase (bet $C$ ), allowing the utilization of this ester as a precursor for glycine betaine production (Østerås et al., 1998). We have previously reported that $H$. elongata can also use choline-O-sulfate as an osmoprotectant (Cánovas et al., 1996), but the mechanism by which this osmoprotection is achieved remains unknown. It is possible that in $H$. elongata choline-O-sulfate does not need to be metabolized to glycine betaine and plays an osmotic role by itself, a situation that has recently been reported for the effective use of choline-O-sulfate as a metabolically inert compatible solute in B. subtilis (Nau-Wagner et al., 1999). However, the absence of a choline sulfatase gene in the Halomonas betIBA region does not rigorously exclude the possibility that choline-O-sulfate could be hydrolysed to choline and then converted into glycine betaine in this moderate halophile. 
By analogy with the corresponding proteins of E. coli (Lamark et al., 1991) and Si. meliloti (Østerås et al., 1998), we conclude that the betI gene product is most probably a regulatory protein. In E. coli, the divergently overlapping bet $T$ and betI promoters are regulated in the same manner by three external stimuli: osmolarity, presence of choline and oxygen. Both promoters remain fully osmotically regulated, but not choline-regulated, in a betI mutant (Lamark et al., 1996). In vivo (Lamark et al., 1996) and in vitro (Røkenes et al., 1996) studies showed that the choline-sensing repressor BetI regulates bet gene expression negatively in response to choline by binding to a 41 bp DNA fragment containing the -10 and -35 regions of both bet promoters. In H. elongata, both the transport and the oxidation of choline to glycine betaine are much faster at high $(2 \mathrm{M} \mathrm{NaCl})$ than at reduced $(0.5 \mathrm{M} \mathrm{NaCl})$ salinity, indicating that osmolarity is a major factor in the regulation of the cholineglycine betaine pathway (Cánovas et al., 1998b). Moreover, the end product glycine betaine exerted a slight inhibition of choline uptake and a considerable inhibition of the oxidation of choline to glycine betaine, especially at high salinity (Cánovas et al., 1998b). Whether or not BetI is involved in these regulatory effects and the role of choline in the regulation of the $H$. elongata bet genes is presently under investigation. Like Si. meliloti (Smith et al., 1988), H. elongata can use both choline and glycine betaine as the sole carbon and nitrogen source (Cánovas et al., 1996), whereas glycine betaine is metabolically inert in E. coli (Landfald \& Strøm, 1986) and B. subtilis (Boch et al., 1994). This requires additional regulatory circuits in $H$. elongata to avoid a futile cycle of glycine betaine biosynthesis and degradation under high osmolarity growth conditions.

To cope with osmotic stress caused by the presence of high salt concentration, halophilic aerobic archaea and the anaerobic bacteria of the order Haloanaerobiales maintain high intracellular salt concentrations. In these extremophiles, enzymes and structural cell components have to be adapted to high salinity and show unique molecular adaptations (Oren, 1999). Examination of the first crystal structures of proteins from the archaeum Haloarcula marismortui suggests that an abundance of acidic residues distributed over the protein surface is a key determinant of adaptation to high-salt conditions (Elcock \& McCammon, 1998). In contrast, halophilic micro-organisms using the compatible solute strategy maintain low salt concentrations within their cytoplasm. Therefore, it is expected that no special adaptation of their intracellular proteins is required. To test this prediction for the BetIBA proteins of the moderate halophile $H$. elongata, their charge and amino acid distribution were analysed and compared with the same proteins of the non-halophilic bacteria E. coli and Si. meliloti. Although the enzymes from Halomonas had a slightly higher percentage of charged amino acids compared to those from E. coli and Si. meliloti, there were no substantial differences in amino acid composition of the Bet proteins from the three microorganisms. BetI proteins showed a relatively high percentage of basic amino acids, with a positive net charge ranging from $9+$ (for Si. meliloti BetI) to $12+$ (for E. coli BetI). Basic and acidic amino acids were present in approximately equivalent numbers in the BetA enzymes of H. elongata (net charge $3+$ ) and E. coli (net charge 1-). However, Si. meliloti BetA was predominantly basic, with a net charge of $13+$. Only BetB exhibited a net negative $(13$ - for the H. elongata enzyme) charge. This holds true for the three BetB enzymes, although BetB from $H$. elongata had a higher negative charge than the enzymes from E. coli $(6-)$ and Si. meliloti $(6-)$. From all these data we conclude that, as far as the betaine synthesis machinery is concerned, no special adaptations to salt seem to exist in the cytoplasmic proteins of the moderate halophile $H$. elongata.

The synthesis of glycine betaine, one of the most powerful osmoprotectants found in nature (Le Rudulier et al., 1984; Csonka \& Hanson, 1991; Kempf \& Bremer, 1998), also plays an important role in the adaptation process of $H$. elongata to a high-osmolarity environment. The bet genes characterized in this work will be used for the construction of single and double mutants affected in the synthesis of glycine betaine and/or other compatible solutes, such as ectoine. These mutants will be of invaluable help in elucidating the global regulation of the osmoadaptive mechanisms in this extremophilic micro-organism.

\section{ACKNOWLEDGEMENTS}

This research was financially supported by grants from the BIOTECH Program of the European Commission (Generic Project Extremophiles as Cell Factories, grant BIO4-CT960488), the Ministerio de Educación y Cultura, Spain (grants PB97-0722 and BIO97-1876-CE), the Junta de Andalucía, the Deutsche Forschungsgemeinschaft through SFB-395, and the Fonds der Chemischen Industrie. D. Cánovas was supported by a fellowship from the Spanish Ministerio de Educación y Cultura and by a short-term fellowship from the EMBO Program.

\section{REFERENCES}

Ausubel, F. M., Brent, R., Kingston, R. E., Moore, D. D., Seidman, J. G., Smith, J. R. \& Struhl, K. (1989). Current Protocols in Molecular Biology. New York: Wiley.

Boch, J., Kempf, B. \& Bremer, E. (1994). Osmoregulation in Bacillus subtilis: synthesis of the osmoprotectant glycine betaine from exogenously provided choline. J Bacteriol 176, 5364-5371.

Boch, J., Kempf, B., Schmid, R. \& Bremer, E. (1996). Synthesis of the osmoprotectant glycine betaine in Bacillus subtilis: characterization of gbsAB genes. J Bacteriol 178, 5121-5129.

Boch, J., Nau-Wagner, G., Kneip, S. \& Bremer, E. (1997). Glycine betaine aldehyde dehydrogenase from Bacillus subtilis: characterization of an enzyme required for the synthesis of the osmoprotectant glycine betaine. Arch Microbiol 168, 282-289.

Brouquisse, R., Weigel, D., Rhodes, D., Yocum, C. F. \& Hanson, A. D. (1989). Evidence for a ferredoxin-dependent choline monooxygenase from spinach chloroplast stroma. Plant Physiol 90, 322-329.

Brow, M. A. D., Pesin, R. \& Sutcliffe, J. G. (1985). The tetracycline repressor protein of pSC101. Mol Biol Evol 2, 1-12. 
Cánovas, D., Vargas, C., Csonka, L. N., Ventosa, A. \& Nieto, J. J. (1996). Osmoprotectants in Halomonas elongata: high-affinity betaine transport system and choline-betaine pathway. J Bacteriol 178, 7221-7226.

Cánovas, D., Vargas, C., Iglesias-Guerra, F., Csonka, L. N., Rhodes, D., Ventosa, A. \& Nieto, J. J. (1997). Isolation and characterization of salt-sensitive mutants of the moderate halophile Halomonas elongata and cloning of the ectoine synthesis genes. J Biol Chem 272, 25794-25801.

Cánovas, D., Vargas, C., Calderón, M. I., Ventosa, A. \& Nieto, J. J. (1998a). Characterization of the genes for the biosynthesis of the compatible solute ectoine in the moderately halophilic bacterium Halomonas elongata DSM 3043. Syst Appl Microbiol 21, 487-497.

Cánovas, D., Vargas, C., Csonka, L. N., Ventosa, A. \& Nieto, J. J. (1998b). Synthesis of glycine betaine from exogenous choline in the moderately halophilic bacterium Halomonas elongata. Appl Environ Microbiol 64, 4095-4097.

Cohen, G. N. \& Rickenberg, R. H. (1956). Concentration specifique reversible des amino acides chez Escherichia coli. Ann Inst Pasteur 91, 693-720.

Csonka, L. N. \& Hanson, A. D. (1991). Prokaryotic osmoregulation: genetics and physiology. Annu Rev Microbiol 45, 569-606.

Dersch, P., Fsihi, H. \& Bremer, E. (1994). Low-copy-number T7 vectors for selective gene expression and efficient protein overproduction in Escherichia coli. FEMS Microbiol Lett 123, 19-26.

Deshnium, P., Los, D. A., Hayashi, H., Mustardy, L. \& Murata, N. (1995). Transformation of Synechococcus with a gene for choline oxidase enhances tolerance to salt stress. Plant Mol Biol 29, 897-907.

Elcock, A. H. \& McCammon, J. A. (1998). Electrostatic contribution to the stability of halophilic proteins. J Mol Biol 280, 731-748.

Göller, K., Ofer, A. \& Galinski, E. A. (1998). Construction of an $\mathrm{NaCl}$-sensitive mutant of Halomonas elongata impaired in ectoine biosynthesis. FEMS Microbiol Lett 161, 293-300.

Guan, K. \& Weiner, H. (1990). Sequence of the precursor of the bovine liver mitochondrial aldehyde dehydrogenase as determined from its cDNA, its genes, and its functionality. Arch Biochem Biophys 277, 351-360.

Haardt, M., Kempf, B., Faatz, E. \& Bremer, E. (1995). The osmoprotectant proline betaine is a major substrate for the binding-protein-dependent transport system ProU of Escherichia coli K-12. Mol Gen Genet 246, 783-786.

Habenicht, A., Hellman, U. \& Cerff, R. (1994). Non-phosphorylating GAPDH of higher plants is a member of the aldehyde dehydrogenase syperfamily with no sequence homology to phosphorylating GAPDH. J Mol Biol 237, 165-171.

Ikuta, S., Imamura, S., Misaki, H. \& Horiuti, Y. (1977). Purification and characterization of choline oxidase from Arthrobacter globiformis. J Biochem 82, 1741-1749.

Johansson, K., El-Ahmad, M., Ramaswamy, S., Hjelmqvist, L., Jörnvall, H. \& Eklund, H. (1998). Structure of betaine aldehyde dehydrogenase at $2 \cdot 1 \AA$ resolution. Protein Sci 7, 2106-2117.

Jones, D. E., Brennan, M. D., Hempel, J. \& Lindalh, R. (1988). Cloning and complete nucleotide sequence of a full-length cDNA encoding a catalytically functional tumour-associated aldehyde dehydrogenase. Proc Natl Acad Sci USA 85, 1782-1786.

Kappes, R. M., Kempf, B., Kneip, S., Boch, J., Gade, J., MeierWagner, J. \& Bremer, E. (1999). Two evolutionary closely related $\mathrm{ABC}$ transporters mediate the uptake of choline for synthesis of the osmoprotectant glycine betaine in Bacillus subtilis. Mol Microbiol 32, 203-216.

Kempf, B. \& Bremer, E. (1998). Uptake and synthesis of compatible solutes as microbial stress-responses to high-osmolality environments. Arch Microbiol 170, 319-330.

Kessler, B., de Lorenzo, V. \& Timmis, K. N. (1992). A general system to integrate $l a c Z$ fusions into the chromosome of gramnegative bacteria: regulation of the $\mathrm{Pm}$ promoter of the TOL plasmid studied with all controlling elements in monocopy. Mol Gen Genet 233, 293-301.

Labes, M., Pühler, A. \& Simon, R. (1990). A new family of RSF1010-derived expression and lac-fusion broad-host-range vectors for gram-negative bacteria. Gene 89, 37-46.

Lamark, T., Kaasen, I., Eshoo, M. W., Falkenberg, P., McDougall, J. \& Strøm, A. R. (1991). DNA sequence and analysis of the bet genes encoding the osmoregulatory choline-glycine betaine pathway of Escherichia coli. Mol Microbiol 5, 1049-1064.

Lamark, T., Røkenes, T. P., McDougall, J. \& Strøm, A. R. (1996). The complex bet promoters of Escherichia coli: regulation by oxygen (ArcA), choline (BetI), and osmotic stress. J Bacteriol 178, 1655-1662.

Landfald, B. \& Strøm, A. R. (1986). Choline-glycine betaine pathway confers a high level of osmotic tolerance in Escherichia coli. J Bacteriol 165, 849-855.

Legaria, J., Rajsbaum, R., Muñoz-Clares, R. A., VillegasSepulveda, N., Simpson, J. \& Iturrriaga, G. (1998). Molecular characterization of two genes encoding betaine-aldehyde dehydrogenase from amaranth. Expression in leaves under shortterm exposure to osmotic stress or abscisic acid. Gene 18, 69-76.

Le Rudulier, D., Strøm, A. R., Dandekar, A. M., Smith, L. T. \& Valentine, R. C. (1984). Molecular biology of osmoregulation. Science 224, 1064-1068.

Liu, Z., Sun, Y., Chung, Y. J. \& 7 other authors (1997). The first structure of an aldehyde dehydrogenase reveals novel interactions between NAD and the Rossmann fold. Nature Struct Biol 4, 317-326.

McCue, K. F. \& Hanson, A. D. (1992). Salt-inducible betaine aldehyde dehydrogenase from sugar beet: cDNA cloning and expression. Plant Mol Biol 18, 1-11.

Miller, J. H. (1972). Experiments in Molecular Genetics. Cold Spring Harbor, NY: Cold Spring Harbor Laboratory.

Miller, J. H. (1991). A Short Course in Bacterial Genetics: a Laboratory Manual and Handbook for Escherichia coli and Related Bacteria. Cold Spring Harbor, NY: Cold Spring Harbor Laboratory.

Nakamura, T., Yokota, S., Muramoto, Y., Tsutsui, K., Oguri, Y., Fukui, K. \& Takabe, T. (1997). Expression of a betaine aldehyde dehydrogenase in rice, a glycine betaine nonaccumulator, and possible location of its protein in peroxisomes. Plant $J$ 11, 1115-1120.

Nau-Wagner, G., Boch, J., Le Good, J. A. \& Bremer, E. (1999). High-affinity transport of choline-O-sulfate and its use as a compatible solute in Bacillus subtilis. Appl Environ Microbiol 65, 560-568.

Oren, A. (1999). Bioenergetic aspects of halophilism. Microbiol Mol Biol Rev 63, 334-348.

Østerås, M., Boncompagni, E., Vincent, N., Poggi, M.-C. \& Le Rudulier, D. (1998). Presence of a gene encoding choline sulfatase in Sinorhizobium meliloti bet operon: choline-O-sulfate is metabolized into glycine betaine. Proc Natl Acad Sci USA 95, 11394-11399.

Pan, W. \& Spratt, B. G. (1994). Regulation of the permeability of 
the gonococcal cell envelope by the $m$ tr system. Mol Microbiol 11, 769-775.

Pocard, J.-A., Vincent, N., Boncompagni, E., Smith, L. T., Poggi, M.-C. \& Le Rudulier, D. (1997). Molecular characterization of the bet genes encoding glycine betaine synthesis in Sinorhizobium meliloti 102F34. Microbiology 143, 1369-1379.

Røkenes, T. P., Lamark, T. \& Strøm, A. R. (1996). DNA-binding properties of the BetI repressor protein of Escherichia coli: the inducer choline stimulates BetI-DNA complex formation. $J$ Bacteriol 178, 1663-1670.

Rosenstein, R., Futter-Bryniok, D. \& Götz, F. (1999). The cholineconverting pathway in Staphylococcus xylosus C2A: genetic and physiological characterization. J Bacteriol 181, 2273-2278.

Saito, Y., Ishii, Y., Hayashi, H. \& 9 other authors (1997). Cloning of genes coding for L-sorbose and L-sorbosone dehydrogenases from Gluconobacter oxydans and microbial production of 2-keto-L-gulonate, a precursor of L-ascorbic acid, in a recombinant G. oxydans strain. Appl Environ Microbiol 63, 454-460.

Sambrook, J., Fritsch, E. F. \& Maniatis, T. (1989). Molecular Cloning: a Laboratory Manual, 2nd edn. Cold Spring Harbor, NY: Cold Spring Harbor Laboratory.

Smith, L. T., Pocard, J. A., Bernard, T. \& Le Rudulier, D. (1988). Osmotic control of glycine betaine synthesis and degradation in Rhizobium meliloti. J Bacteriol 170, 3142-3149.

Steinmetz, C. G., Xie, P., Weiner, H. \& Hurley, T. (1997). Structure of a mitochondrial aldehyde dehydrogenase: the genetic component of ethanol aversion. Structure 5, 701-711.
Takeshita, S., Sato, M., Tabo, M., Masahashi, W. \& HashimotoGothoh, T. (1987). High-copy-number and low-copy-number plasmid vectors for lac $Z \alpha$-complementation and chloramphenicol- or kanamycin-resistance selection. Gene 61, 63-74.

Vargas, C., Coronado, M. J., Ventosa, A. \& Nieto, J. J. (1997). Host range, stability and compatibility of broad host-range-plasmids and a shuttle vector in moderately halophilic bacteria. Evidence of intragenic and intergenic conjugation in moderate halophiles. Syst Appl Microbiol 20, 173-181.

Ventosa, A. \& Nieto, J. J. (1995). Biotechnological applications and potentialities of halophilic microorganisms. World J Microbiol Biotechnol 11, 85-94.

Ventosa, A., Nieto, J. J. \& Oren, A. (1998). Biology of moderately halophilic aerobic bacteria. Microbiol Mol Biol Rev 62, 504-544.

Vreeland, R. H. (1992). The family Halomonadaceae. In The Prokaryotes, 2nd edn, pp. 3181-3188. Edited by A. Balows, H. G. Trüper, M. Dworkin, W. Harder \& K. H. Schleifer. New York: Springer.

Weretilnyk, E. A. \& Hanson, A. D. (1990). Molecular cloning of a plant betaine-aldehyde dehydrogenase, an enzyme implicated in adaption to salinity and drought. Proc Natl Acad Sci USA 87, 2745-2749.

Wierenga, R. K., Terpstra, P. \& Hol, W. G. J. (1986). Prediction of the occurrence of the ADP-binding $\beta \alpha \beta$-fold in proteins, using an amino acid sequence fingerprint. J Mol Biol 187, 101-117.

Received 30 July 1999; revised 18 October 1999; accepted 2 November 1999. 